\title{
Minocycline: a novel stroke therapy
}

\begin{abstract}
The only FDA approved treatment for ischemic strokes is tissue plasminogen activator (tPA). The benefit of treatment with tPA is limited and it has to be administrated within a short time window; furthermore, the side effects are serious including increased risk of subsequent hemorrhagic transformation. This review discusses minocycline's role in neuroprotection, and it discusses the results of five published clinical trials regarding the use of administration of minocycline for treatment of acute stroke. The studies find that the benefit of tPA treatment alone is limited and has serious side effects. While many new agents have been studied to ameliorate this, minocycline, a tetracycline antibiotic, seems the most promising. Some investigators have suggested that administration of minocycline may be suitable as a general treatment of patients with acute stroke that is safe and can be used in both ischemic and hemorrhagic strokes as a single treatment that may provide benefits in reducing the deficits from ischemic stokes with little side effects.
\end{abstract}

Volume 2 Issue 6 - 2015

\author{
Shyam Vedantam, Aage R Moller \\ The University of Texas at Dallas School of Behavioral and Brain \\ Sciences, USA
}

Correspondence: Aage R Moller, School of Behavioral and Brain Sciences, The University of Texas at Dallas, GR 4I, $800 \mathrm{~W}$ Campbell Road, Richardson, TX 75080, USA, Email amoller@utdallas.edu

Received: July 13,2015 | Published: September 10, 2015

Keywords: Stroke, Minocycline, Neuroprotection, tPA

\section{Introduction}

Strokes are a cause of death in western, industrialized countries and are a major cause of long-lasting disability. Approximately 795,000 new and recurrent ischemic or hemorrhagic strokes occurred each year from 2000 to 2010 in the United States. Of these approximately 610,000 were first events and 185,000 were recurrent strokes. ${ }^{1}$ More than 690,000 ischemic strokes have been reported to occur every year in the USA. ${ }^{2}$ Stroke is more frequent in men than in women, of all ages. Up to $12 \%$ of people of age 45-64 dies within 30 days after an ischemic stroke. ${ }^{3,4}$ One of every 19 deaths in the USA is caused by strokes. Thrombolytic therapy of acute ischemic strokes with tPA is the only FDA approved therapy, but because of the adverse side effects from treatment with tPA, many physicians have opted out of treating stroke patients with tPA. There is therefore a need for an improved stroke therapy that can be used inside a larger time window, is safer to use, perhaps can be used in combination with tPA to extend the latter's use, and give better long-term outcomes by saving the penumbra and restore neural function.

Great progress has been made in finding the risk factors for stroke. ${ }^{2,5}$ It was estimated that $40 \%$ of the patients who had ischemic stroke would have significant functional disability or death following stroke. This is only little changed despite the progress in determining the risk factors for ischemic stroke. Control of blood pressure and some supplements such as magnesium have been found to reduce the risk of stroke.

Many compounds have been investigated to reduce infarct size and improve neurological outcomes, and some have shown effect in animal models. Yet, most of these compounds have failed in the transition to clinical, due to deleterious side effects or an even more limited time window.

Studies have suggested various forms of anti-inflammatory treatments to reduce the deficits from strokes. It is a problem; however, that inflammation may be both beneficial and destructive depending on the timing of the intervention of the inflammation modulation.

In this review we discuss the use of a readily available substance, minocycline, for reducing the harm from inflammatory processes that occurs subsequent to the acute phase of an ischemic stroke. This review also discusses the neuroprotective properties of minocycline.

\section{Nature of the pathology of ischemic strokes}

The ischemic insult from stroke causes necrotic cell death by changing the balance in $\mathrm{pH}$, nutrition, waste removal, and temperature. A subsequent ischemic cascade occurs. It was mentioned by Mass \& Sadieh, ${ }^{6}$ that $70 \%$ of the metabolic demand in the brain is due to the sodium-potassium ATPase pump that maintains ion gradient necessary for cell function. Immediately after blocking blood supply to a region of the brain a cascade of events occurs, causing cells to rapidly depolarize. Glutamate release leads to calcium influx through NMDA receptors, which in turn leads to excitotoxic cell death. As described by Gotoh et al. ${ }^{7}$ ischemia subsequently breaks down blood-brain barrier hours after infarction, causing vasogenic edema. Depolarization waves spread from the infarct core, damaging surrounding cells, eventually involving the penumbra.

There is evidence that the destruction of brain tissue from ischemia somehow causes an immune reaction and that the resulting inflammatory reactions cause some of the acute damage and, in particular, contribute to the progression of the damage that was caused by the initial localized ischemia. ${ }^{8}$ This means that treatment that somehow reduces the inflammatory processes induced by activation of the innate or the adaptive immune system might reduce the deficits from strokes.

A review by Wang et al. ${ }^{9}$ has described the inflammatory response in stroke. Reperfusion of an occluded vessel leads to a generation of reactive oxygen species (ROS). ROS then stimulate ischemic cells to secrete inflammatory cytokines and chemokines that upregulate adhesion molecules and peripheral leukocyte recruitment, respectively. ${ }^{9}$ Activated inflammatory cells also release more cytokines, matrix metalloproteinases (MMPs), nitric oxide, and more ROS. Secretion of MMPs lead to blood-brain barrier disruption, which exacerbates the injury through involvement of activated microglia and involved peripheral inflammatory cells. Importantly though, Wang ${ }^{10}$ concluded that while inflammation is an important mediator in the pathology of ischemic stroke, inflammation may play both beneficial and detrimental role: Wang ${ }^{10}$ in 2003 suggested that early inflammatory responses may potentiate injury while later responses might be important in recovery and repair 


\section{Treatment of ischemic stroke}

The only FDA approved treatment of acute strokes is thrombolytic therapy using tissue plasminogen activator (tPA) which has a limited time window for administration, ${ }^{11}$ and its beneficial effect is limited. The use tPA therapy in patients with ischemic stroke was approved by the FDA $1996 .{ }^{2}$ Therefore considerable experience of its use has been gained.

tPA therapy has several side effects and severe limitations. It increases the risk of bleeding and can therefore not be used in treatment of hemorrhagic stroke, and treatment of ischemic stroke with tPA may cause transformation into hemorrhagic stroke. Therefore, tPA cannot be administrated before it is ruled out that the stroke is not a hemorrhagic stroke. Consequently, a CT must be performed, which delays therapy with tPA. A medical history of recent brain trauma or surgeries, bleeding problems, uncontrolled high blood pressure or use of blood thinners rules out tPA use. Due to the nature of hospital organization, these studies may take several hours. Add to this the time it takes for a person to reach the hospital, several hours can elapse before treatment can be made. The upper time limit for delay from the occurrence of a stroke and the administration of tPA is regarded to be 3 to 4.5 hours.

A study by The National Institutes of Neurological Disorders and Stroke (NINDS) found that the neurological functioning statistically increased at 30 days post-stroke in patients who had received tPA against those who had not. ${ }^{12}$ The study did concede though that giving tPA in acute ischemic stroke within the first 180 minutes did not yield a statistically significant benefit in the first 24 hours and even increased the risk of hemorrhagic stroke.

\section{Actions of tPA}

tPA binds to the fibrin on the clot surface, activating fibrinbound plasminogen and cleaving plasmin from this bound complex, which goes on to break apart the collected fibrin strands of the clot. ${ }^{13}$ Circulating $\alpha 2$-antiplasmin inactivates this plasmin, so that a natural balance may be maintained in the body. To override this limiting effect, intravenous tPA is administered. tPA can also increase the permeability of the blood-brain barrier from activation of latent platelet-derived growth factor-CC (PDGF-CC), ${ }^{14,15}$ and an increase in matrix metalloproteinases. ${ }^{16}$ Furthermore, tPA enhances the excitotoxic effect of glutamatergic (NMDA) receptors. ${ }^{17}$

\section{Minocycline}

Minocycline is a semisynthetic drug that has been in use since the 1970s. It is in the tetracycline family of antibiotics, which were discovered in 1948 by Duggar ${ }^{18}$ as a natural product of Streptomyces. Streptomyces are soil-dwelling bacteria that produce tetracycline among other diverse products. Modifications to the chemically isolated byproducts eventually led to two of the more common semi-synthetic tetracycline used clinically today as antibiotics, doxycycline and minocycline. In order to exert this activity, tetracycline inhibits protein translation by binding to the P30 subunit of the ribosome.

Minocycline is distinguishable from other tetracycline for its highly lipophilic nature; it is a superior blood-brain barrier penetrator compared to the other tetracycline, including doxycycline. ${ }^{19}$ It has properties, discussed below, that are outside of its antibiotic abilities.

\section{Pharmacolkinetics}

Recent studies indicate that minocycline's therapeutic window show is wide enough for clinical application. $.^{20} \mathrm{~A} 3 \mathrm{mg} / \mathrm{kg}$ intravenous dose of minocycline yielded similar to plasma peak concentrations of $200 \mathrm{mg}$ oral dose in humans and successfully reduced infarct size in ischemic stroke. ${ }^{21}$

\section{Minocycline for neuroprotection}

The precise target of minocycline's neuroprotection in acute stroke is unknown. Minocycline has been shown to alter many cellular processes that may have beneficial relevance to stroke recovery.

Programmed cell death, or apoptosis, is largely mediated by caspases. The two pathways of caspase activation, extrinsic and intrinsic, are named after the source of the stress to the cell. In the intrinsic pathway, different variables inevitably lead to mitochondrial outer membrane permeabilization, causing decreased ATP production, and further release of proteins that lead to caspase activation. Some of these include cytochrome c, apoptosis-inducing factor (AIF), and direct IAP-binding protein with low PI (DIABLO/SMAC) among others. The extrinsic pathway involves DNA damage that leads to over-activation of PARP, release of AIF, and endonuclease G, all of which leads to apoptosis. ${ }^{22}$

The Bcl-2 family of proteins is involved in the pathway of intrinsic activation; however, there are pro-apoptotic and antiapoptotic members of this family. By inducing anti-apoptotic Bcl-2 proteins and inhibiting cytochrome c release, minocycline has been shown to protect kidney epithelial cells at the mitochondrial level. ${ }^{23}$ Minocycline showed similar results in neurons (but not astrocytes), as well as improved stroke-induced behavioral effects and cerebral infarction areas, but showed a dose-dependent effect in that high doses exacerbated ischemic injury. ${ }^{24}$

\section{Reactive oxygen species scavenging}

The initial occlusion of a blood vessel in ischemic stroke damages tissue by inadequate perfusion. When blood flow is reestablished, either spontaneously or by therapeutic intervention, the affected tissue may undergo reperfusion injury. This is associated with deleterious effects in both stroke and myocardial infarctions. ${ }^{25}$ The surge of oxygen into these injured tissues leads to generation of reactive oxygen species (ROS). This, in turn, causes mitochondrial permeability transition which leads to mitochondrial depolarization and swelling, along with outer membrane rupture which leads to cytochrome $\mathrm{c}$ release and subsequent activation of caspases to end in cell death.

Minocycline's ability to protect against oxidative stress may be due to its activity as a ROS scavenger. Minocycline's direct ROS scavenging is due to its multiply substituted phenol ring resulting in a phenol-derived free radical that is stable and unreactive due to resonance stabilization and number and size of the other phenol ring substituents. Minocycline was at least 200 -fold better at ROS scavenging than tetracycline; ${ }^{26}$ these authors attribute this to minocycline's dimethylamino substituent on the phenol ring, which provides for increased resonance and steric stabilization.

\section{Inhibition of microglia}

Minocycline is a known inhibitor of microglial activity in the brain. ${ }^{27}$ Early research showed that minocycline prevented the excitotoxin-induced proliferation of microglia. ${ }^{28}$ Minocycline also reduced the microglia-induced cell death of endothelial cells and astrocytes by half. ${ }^{29}$ Though the microglial M1/M2 switch has not been thoroughly studied, recently published work has indicated that early minocycline treatment promoted neurovascular remodeling post-stroke and promoted M2-phenotype microglia activation even at 4 weeks post-stroke. ${ }^{30}$ 


\section{Suppression of MMPs}

MMP-2 has been shown to play a role in early blood-brain barrier disruption. ${ }^{31}$ Furthermore, direct injection of MMP-2 onto the rodent brain disrupts the blood-brain barrier. ${ }^{32}$ While this does provide evidence for MMP-2's roles in hemorrhagic transformation, its role in ischemia is less certain. Knockout of MMP-2 did not reduce infarct size in a 2-hour MCAO model in mice, but this study did find that MMP-9 levels were increased. ${ }^{33}$

Animal models have shown that MMP-9 is actively induced following ischemia, and is localized to endothelial cells and parenchymal cells. Knockout or inhibition of MMP-9 has been shown to decrease lesion volume. ${ }^{33}$ One review has suggested that early hemorrhagic transformation ( $<24$ hours after ischemic stroke) occurs due to leukocyte-derived MMP-9 and brain-derived MMP-2, while delayed hemorrhagic transformation ( $>24$ hours after ischemic stroke) are related to ischemic activation of brain proteases MMP-2, MMP-3, and MMP-9, neuroinflammation, and factors that promote vascular remodeling. ${ }^{34}$

Many studies have found that Matrix Metalloproteinases (MMPs) plays a critical role during human stroke, especially MMP-9. Castellanos et $a 1 .{ }^{35}$ found that elevated MMP-9 in the acute phase of a cerebral infarct was indicative of hemorrhagic transformation. Montaner et al. ${ }^{36}$ found that even baseline MMP-9 levels predicted hemorrhagic transformation after tPA treatment. Both MMP-2 and MMP-9 are elevated after cerebral infarct, and MMP-9 levels were increased in patients treated with tPA compared to those treated with hypothermia. MMP-9 levels are higher in brain tissue in both human ischemic and hemorrhagic stroke cases. ${ }^{37}$

Intraperitoneal treatments of minocycline in rats significantly reduced activity and protein concentration of both MMP-2 and MMP9, and MMP-9 was extremely sensitive even at low doses. ${ }^{38}$

\section{Treatment with minocycline in humans}

Neuroprotection is the term used for actions that protect the integrity of the central nervous system in cases of ischemic damage such as from ischemic stokes of other forms of trauma. ${ }^{39}$ The interest into minocycline as a potential neuroprotective agent in stroke coalesced with the landmark studies by Yrjänheikki. ${ }^{40}$ In the first of their studies, they found that minocycline was neuroprotective in a model of global ischemia. ${ }^{41}$ In the second, minocycline was shown to reduce infarct volume when given before and after transient middle cerebral artery occlusion (MCAO).$^{40}$ This sparked studies into minocycline's potential as a therapeutic agent in ischemic stroke.

Treatment with minocycline is different from treatment with tPA. It does not affect coagulations and it can therefore be administered without ensuring that the patient does not have a hemorrhagic stroke. It is an antibiotic of the tetracycline family and its beneficial effect for stroke is related to its anti-inflammatory and neuroprotective action. Minocycline it is not approved for treatment by FDA for treatment of stroke. It can be used as an "off-label" treatment.

There is conflicting data on the use of minocycline in hemorrhagic stroke. In one study, minocycline was found to reduce MMP levels, especially MMP-12, and some positive behavioral outcomes on days 7 and 28 after stroke. ${ }^{42} \mathrm{~A}$ more recent study found that while minocycline did suppress microglial and macrophage activation in peri-infarct region, there was no reduction in infarct volume or functional benefit on days 5 or 14 post-intracerebral hemorrhage. ${ }^{43}$ However, there is limited research done in this area and more will need to be done to elucidate minocycline's neuroprotective ability in hemorrhagic stroke. Minocycline can be used without adverse side effects in the acute stroke period and perhaps throughout the subsequent recovery period.

Early research has shown that acute administration of minocycline up to 5 hours after ischemic insult reduces infarct size and inflammation in animal models. ${ }^{44}$ Another study correlated these results in human trials, finding that minocycline was effective at reducing infarct size when administered 5 hours after ischemic insult. ${ }^{21}$

On a longer time scale, two studies found that minocycline improved neurological functioning and behavioral recovery when given for four days after ischemic insult in MCAO rodent model and then for four weeks. ${ }^{45}$ In the second animal study, ${ }^{46}$ minocycline did not reduce infarct size, but did decrease the number of activated microglia while preserving plasticity in the dentate gyrus.

Recently published clinical trials varied on whether they gave minocycline orally or intravenously, the dose used, and how often minocycline was administered. In 1988, Saivin and Houin ${ }^{19}$ described the pharmacokinetics of minocycline. The $200 \mathrm{mg}$ dose, which is the standard used in many of the studies conducted, yields a peak concentration of $6 \mathrm{mg} / \mathrm{L}$ in humans. The steady state concentration achieved when this dose is split in half and given orally is $1.4-1.8 \mathrm{mg} /$ L. As aforementioned, minocycline is highly lipophilic and penetrates the blood-brain barrier well. As such, the CSF concentration is approximately $0.5 \mathrm{mg} / \mathrm{L}$. In humans, the half-life is approximately 24 hours, ${ }^{21}$ while in rats; it is 3 hours. ${ }^{47}$

\section{Tolerability}

When taken chronically, patients taking above 100mg daily commonly complained of dizziness. ${ }^{48}$ However, in acute therapy in an exploratory clinical trial of stroke patients, minocycline was found to be well tolerated up to $10 \mathrm{mg} / \mathrm{kg} \mathrm{IV}{ }^{21}$ Even when used at doses from $200-400 \mathrm{mg}$ (IV) and given from 2-24 days, only $18 \%$ of patients complained of side effects, with half involving gastrointestinal complaints ${ }^{49}$ From a tolerability standpoint, there is no issue for its use as an acute ischemic stroke therapy.

\section{Results of clinical trials of treatment with minocycline}

There are currently five published clinical trials evaluating the use of minocycline in acute ischemic stroke (in order of publication date): Minocycline treatment in acute stroke; ${ }^{50}$ Minocycline to improve neurologic outcome in stroke: a dose-finding study; ${ }^{21}$ Efficacy of minocycline in acute ischemic stroke: A single-blinded, placebo-controlled trial; ${ }^{51}$ Intravenous minocycline in acute stroke: a randomized, controlled pilot study and meta-analysis; $;{ }^{52} \mathrm{An}$ open-label evaluator-blinded clinical study of minocycline neuroprotection in ischemic stroke: gender-dependent effect. ${ }^{53}$

Lampl et al. ${ }^{50}$ did a randomized, open-label, evaluator-blinded study of minocycline administered to 152 patients with acute ischemic stroke age $\geq 18$, NIHSS(NIH Stroke Scale) score greater than 5 , and stroke onset 6-24 hours before treatment, $35.1 \%$ female; mean age $66.7 \pm 11.11$ years). Average time to treatment in minocycline group was 12.64 hours compared to 11.99 hours in placebo group. They compared NIH stroke scale (NIHSS) scores, which are used to measure stroke-related neurologic deficits (higher scores indicative of more severe symptoms) on day 90 in patients either treated with or without $200 \mathrm{mg}$ /day oral minocycline. They also included NIHSS score on days 7 and 30, modified Rankin Scale (mRS) scores (measure of the degree of disability regarding daily activities) on 
days 7,30 , and 90 to measure degree of disability in daily activities (lower score indicative of less severe disability), and Barthel Index (BI) scores (measure of performance in activities of daily living) on those same days to measure for performance in activities of daily living (higher score indicative of better performance). Patients with a history of hemorrhagic stroke were excluded as were people with other central nervous system disease, tPA use, pre-existing neurologic disability, known allergy to tetracyclines, acute or chronic renal failure, pre-existing infectious disease requiring antibiotic therapy, and swallowing difficulties.

The mean NIHSS scores between the minocycline-treated group and control group were similar ( $7.5 \pm 3.2$ vs $7.6 \pm 3.8$, respectively). There was a significant difference at day 90 between the two groups as the minocycline-treated group had a significantly improved NIHSS score compared to the control group ( $1.6 \pm 1.9$ vs $6.5 \pm 3.8$, respectively). Improvement in treatment vs. no treatment groups was seen on day 7. The secondary outcomes showed significant differences between treatment and control groups at days 7, 30, and 90.

Fagan et al. ${ }^{21}$ performed an open-label, dose-escalation trial in 60 patients older than 18 (age: $65 \pm 13.7$ years; race: $83 \%$ white; sex: $47 \%$ female) who had acute onset of focal neurologic deficit consistent with acute ischemic stroke or computed tomographic scan consistent with acute cerebral ischemia, symptom onset less than 6 hours, and NIHSS score greater than 1. tPA was also given in $60 \%$ of the patients. NIHSS score at baseline was $8.7 \pm 5.8$. The mean time from symptom onset to IV minocycline infusion was $307.4 \pm 50$ minutes. The primary objective was to identify the safety of giving minocycline intravenously to patients with acute ischemic stroke, in combination with tPA and within 6 hours of symptom onset. Four dose tiers were tested: $3 \mathrm{mg} / \mathrm{kg}, 4.5 \mathrm{mg} / \mathrm{kg}, 6 \mathrm{mg} / \mathrm{kg}$, and $10 \mathrm{mg} / \mathrm{kg}$. Treatment with tPA was allowed. No control was used. Patients were given an IV loading dose of minocycline followed by maintenance doses of half the daily dose every twelve hours for a total of six doses. Inclusion criteria were patient.

Exclusion criteria were similar to those in the study by Lamp et al ${ }^{50}$ but also included severe stroke (NIHSS $>22$ ), pregnancy, thrombocytopenia, dizziness at time of stroke or in the past month, aphasia, availability for 90-day follow-up, and those who underwent interventional neuroradiological intervention in the first 12 hours.

At 90 days, half of the patients had a mRS of 0 or 1 , with no difference between dose tiers (again, no control used). Only one case of dose limiting toxicity was found, and this was at the highest dose administration (1/41 patients at this level). The participants reported that side effects reported were mild, self-limiting, and unrelated to dose (all being reversible). No cases of thrombophlebitis were reported. Those patients given tPA did not have subsequent severe intracerebral hemorrhage, and minocycline was well tolerated at all doses.

Padma-Srivastava et al. ${ }^{51}$ reported the results of investigated the efficacy of minocycline in 23 patients with first-ever acute ischemic stroke 6-24 hours of symptom onset in a single-blinded, placebocontrolled study conducted at the All India Institute of Medical Sciences. The mean age was $52.7 \pm 15.3$ year and male: female ratio of 13:10 compared with a control group of 27 patients with acute ischemic stroke with mean age of $57 \pm 14.2$ and male: female ratio of 18:9. All participants were given $200 \mathrm{mg}$ capsule of minocycline once a day for five days orally (or through Ryle's tube if they had difficulty swallowing) or oral vitamin B capsules as a placebo. The mean time to treatment in minocycline group was $13.5 \mathrm{~h}$ and, in the control group, it was $11.99 \mathrm{~h}$.
No mortality, myocardial infarctions, recurrent strokes, and hemorrhagic transformations were noted in either group.

There was no significant difference between treatment and control groups at days one and seven, but NIHSS scores improved an average of two points versus the control cohort at the primary endpoint of 90 days, with improvement showing at 30 days as well. mRS score (1.8 vs 2.4$)$ and $\mathrm{mBI}(75.3$ vs 64.1$)$ also improved over the threemonth time period in the treatment group versus the control group, respectively.

Kohler et al. ${ }^{52}$ performed a multicenter, prospective, randomized open-label, blinded, end point evaluation pilot study of the effect of administration of minocycline to patients with acute stroke that had enrolled 95 patients. Exclusion criteria were similar to the Fagan et al. ${ }^{21}$ study. This study, done at three hospitals in Perth, Western Australia, included patients who had both ischemic and hemorrhagic stokes and those given and not given TPA, although both represented small subgroups of the overall study. The primary endpoint was mRS $\leq 2$ at day 90 for patients given or not given $100 \mathrm{mg}$ minocycline IV every 12 hours for a total of five doses. Secondary outcomes included NIHSS at day 7 and BI at day 90 . Inclusion criteria were age great than (or equal to) 18 , onset of stroke within 24 hours, any measurable NIHSS scale deficit, and ability to provide informed consent.

The two groups did not differ in age $(67.7 \pm 11.0$ years in treatment vs $67.9 \pm 16.3$ years in control), sex $(38.3 \%$ female in treatment vs $43.8 \%$ female in control), mean NIHSS score $(9.1 \pm 7.2$ in treatment group vs $8.7 \pm 6.4$ in control) or other baseline characteristics. The mean time to treatment was 10.7 hours in minocycline group (14 patients between 0-6 hours; 15 between $6-12 ; 6$ between 12-18; 9 between $18-24)$. Hemorrhagic stroke was present in 5 patients (11.4\%) given minocycline and 6 patients $(12.5 \%)$ in control. Thrombolysis with tPA was given in 8 patients (18.2\%) given minocycline and 6 patients $(12.5 \%)$ in control. The primary endpoint was $\mathrm{mRS} \leq 2$, and Kohler et al. ${ }^{52}$ reported no significant difference in the primary and secondary outcomes between the two groups; $65.9 \%$ of the minocycline-treated group and $70.2 \%$ of the control group reached this goal.

Analysis of the categorical shift in mRS at day 90 and secondary outcomes showed similar results, per Kohler et al. ${ }^{52}$ The study did find similar numbers of death ( $4.2 \%$ both treatment and control groups) and hemorrhagic transformation (6.2\% in treatment and $4.2 \%$ in control) in both groups. Urinary tract infections occurred in no patients given minocycline and 7 patients (14.6\%) in control group. The study had 95 participants divided into two groups, one with 47 participants to receive minocycline and another with 48 participants to receive no minocycline. In the intention-to-treat population, 29 of $47(65.9 \%)$ allocated minocycline survived free of handicap compared with 33 of $48(70.2 \%)$ allocated no minocycline (rate ratio, $0.94 ; 95 \%$ confidence interval, $0.71-1.25$ and odds ratio, 0.73 ; 95\% CI, 0.31-1.71). A metaanalysis of the 3 human trials suggests minocycline may increase the odds of handicap-free survival by 3 -fold (odds ratio, 2.99; 95\% CI, 1.74-5.16) but there was substantial heterogeneity among the trials. ${ }^{52}$

In this pilot study of a small sample of acute stroke patients, intravenous minocycline was regarded to be safe but not efficacious. The authors of the study admitted that it was not powered to identify reliably or exclude a modest but clinically important treatment effect of minocycline. Larger trials would improve the precision of the estimates of any treatment effect of minocycline. They state the negative efficacy result of our study may be a false-negative (i.e. a type II error) because of inadequate statistical power, and minocycline could potentially improve survival free of handicap by as much as $25 \%$ (i.e. the upper $95 \% \mathrm{CI}$ of the observed estimate of the rate ratio, $0.94 ; 95 \%$ CI, 0.71-1.25). 
An open-label, evaluator-blinded clinical trial by Amiri-Nikpour et al..$^{53}$ is the latest study of the results of administration of minocycline to patients with acute ischemic stroke. Similar criteria were used for inclusion as in the Lampl study. Onset of symptoms was between 6-24 hours, NIHSS scores greater than 5, and computed tomography compatible with an acute stroke. Exclusion criteria were similar to the Lampl et al. ${ }^{54}$ study (including tPA use), as well as pregnancy and estrogen therapy. 60 patients were divided into a control group and a minocycline-treated group, which was given $200 \mathrm{mg}$ of oral minocycline once daily for 5 days from symptom onset. Both control and treatment groups were given 100mg aspirin daily. All participants received therapy within 9 hours of arriving at the hospital. The primary end points were NIHSS scores at day 90, but were also collected on days 30 and 60 .

Seven patients dropped out of the study, so only 53 were analyzed (26 in treatment group; 27 in control group). The mean age was similar between the treatment and control groups $(65.23 \pm 9$ vs $66.52 \pm 7.8$ years, respectively). Females were evenly distributed between the two groups $(53.8 \%$ in treatment vs $51.9 \%$ in control, $\mathrm{P}=0.884)$. No significant differences in risk factors between the two groups were detected by the research group, though they do report that only the control group had taken antihypertensive and anti-diabetic drugs. Per Amiri-Nikpour et al..$^{53}$ there was no significant difference in stroke location between the two groups.

Fifty-three patients $(88.3 \%)$ completed the study. The NIHSS score at admission was 9 amongst both groups. Among all patients, NIHSS score was significantly lower in the minocycline-treated compared with control on day 90 (minocycline median 4 , interquartile range 4-7, control median 7, interquartile range $5-8, \mathrm{P}=0.031$ ). While the NIHSS score only improved 2 points for the control group at day 90 , the NIHSS score improved by 5 points for the treatment group. The study related that there was no significant difference between the two groups, in terms of NIHSS score, at days 30 and 60.

When the participants were divided into sex subgroups for further analysis, the aforementioned improvement in NIHSS score held significant for male participants and was present on days 30 and 60 as well. Analysis of female subgroups showed that NIHSS score at baseline for the minocycline-treated group was actually higher than the NIHSS score for the control group. In addition, while the NIHSS scores in both groups improved, there was no statistically significant difference between the improvement in the treatment and control groups. No adverse outcomes including myocardial infarction, recurrent stroke, or mortality were observed during the study.

The results of the five studies are summarized in Table 1.

\section{Discussion}

The results of the published clinical trials of the effect of administration of minocycline to patients with acute strokes are encouraging although some of the studies had several limitations. The studies' independent statistical analyses showed that minocycline was effective in improving neurological outcome. All but one found that their primary endpoints were achieved --NIHSS scores at day 90 were statistically improved in the minocycline group in the Lampl et al. ${ }^{50}$ Padma-Srivastava et al. ${ }^{51}$ and Amiri-Nikpour et al. ${ }^{53}$ studies, and the Fagan et al. ${ }^{21}$ study found minocycline to be well tolerated at their dose range with and without tPA. The only study that didn't find their primary endpoint reached, which was a $\mathrm{mRS} \leq 2$ at day 90 , was the Kohler et al. ${ }^{52}$ study.

Many also found their secondary endpoints to also be achieved. The Lampl et al..$^{50}$ study found NIHSS scores, mRS, and BI scores decrease significantly over time in the minocycline-treated group vs the control group. Fagan et al. ${ }^{21}$ found that half of their patients had a mRS of 0 or 1 at day 90 . Padma-Srivastava et al..$^{51}$ found that NIHSS score improved even at day 30 , and the $\mathrm{mRS}$ and $\mathrm{mBI}$ scores significantly decreased over the three month period in the minocycline treated group. In contrast, the Kohler et al. ${ }^{52}$ study found no statistically significant difference in the NIHSS and BI at the other time points they were recorded. Similarly, Amiri-Nikpour et al. ${ }^{53}$ study found that there was no statistically significant difference in NIHSS score at days 30 or 90 between the treatment or control groups.

In the 5 published clinical trials of minocycline in acute ischemic stroke, minocycline use was shown to improve neurological functioning on any of the measures used to quantify neurological improvement after acute ischemic stroke. While minocycline might not show immediate improvement on day 1, this data does show that improvement can be seen as soon as three months post-stroke. Studies that had long times between stroke onset and administration of minocycline showed similar results. In three studies the time between stroke onset and administration of minocycline ranged from 10-13.5 average hours. Only in one study, the Fagan et al. ${ }^{21}$ was the average time before administration of minocycline 6 hours or less.

Only one study, done by Kohler et al..$^{52}$ did not show efficacy of minocycline in treating acute stroke. However, it should be noted that the dose used in this study was only $100 \mathrm{mg} /$ day, half of what the other studies used, and was continued only for 2.5 days. As other groups found minocycline to have some therapeutic benefit at $200 \mathrm{mg} /$ day orally for 5 days, this seems appropriate for future studies and applications (though $3 \mathrm{mg} / \mathrm{kg}$ IV causes the same plasma concentration and avoids any dysphagia complications).

Two studies also employed adjunct tPA, but in one more than the other. The Fagan et al. ${ }^{21}$ study found that even though $60 \%$ of the patients were given tPA on top of minocycline, none of them had severe subsequent hemorrhagic stroke. In the Kohler et al..$^{52}$ study, 1 of 8 patients given minocycline and tPA had subsequent hemorrhagic stroke, and this ratio was similar in the tPA-only group.

The limitations of the clinical trials include their relatively small sample size. Only one study, done by Fagan et al. ${ }^{21}$ had more than 100 patients. Two studies, Amiri-Nikpour et al..$^{53}$ and Padma-Srivastava et al. ${ }^{51}$ only had about 50 patients, while Fagan et al. ${ }^{21}$ had 60 and Kohler et al. ${ }^{52}$ had 95 participants. These studies were too small to ensure statistical rigor. Three studies (Amiri-Nikpour et al..$^{53} \mathrm{Lampl}$ et al. ${ }^{50}$ and Kohler et al. ${ }^{52}$ ) were designed as open-label studies, which could have led to investigative bias, however unlikely.

In addition, route of administration varied between the studies. Three out of the five studies gave oral minocycline doses instead of through an IV route. Oral administration of minocycline leads to absorption of about $95-100 \%$ through the stomach and small intestine. ${ }^{54}$ However, peak concentrations when given orally are achieved at up to 3 hours of administration orally $y^{55}$ this delay, as compared to IV administration, may limit minocycline's efficacy in acute stroke, where time is of the essence. The Fagan et al. ${ }^{21}$ study found that IV doses up to $10 \mathrm{mg} / \mathrm{kg}$ were mostly safe, with only one dose-limiting case. Since oral minocycline may not be easily swallowed following a stroke, IV administration represents a more optimal route. Furthermore, duration of administration varied in one trial --the only published study to find that minocycline was ineffective also used a shorter duration of administration ( 2.5 versus 5 days) ${ }^{52}$

The studies by Amiri-Nikpour et al..$^{53}$ and Fagan et al. ${ }^{21}$ also did not use a placebo as a control (the Amiri-Nikpour et al. ${ }^{53}$ study 
used administration of $100 \mathrm{mg}$ aspirin as the control). While the Amiri-Nikpour et al. ${ }^{53}$ studies cites a meta-analysis that suggested no superiority of placebo over no-treatment in clinical trials existed when the outcome had been an objective measurement, ${ }^{56}$ this study design could have led to the false-negative effect found (no benefit for females).

Only two studies, Fagan et al. ${ }^{21}$ and Kohler et al. ${ }^{52}$ allowed for adjunct tPA use, though the latter did admit that they failed to account for the potential beneficially effects of combination treatments. Combining tPA and minocycline could lead to a synergistic effect (see below).

Finally, the time between stroke onset and time of medication administration is lackluster in most of these studies. For reference, tPA must be given within the first 3-4.5 hours of stroke. In this study, the time frame ranged from 5 hours to 24 hours. The Fagan et al. ${ }^{21}$ Padma-Srivastava et al. ${ }^{51}$ and Kohler et al. ${ }^{52}$ studies averaged administration of minocycline about 10-12 hours after stroke onset. Cells start exhibiting DNA fragmentation as early as 30 minutes after stroke onset, with peak at 24-48 hours and persistence for 4 weeks. ${ }^{39}$ Therefore, it is imperative that faster diagnosis of stroke and initiation of treatment protocol be administered. This will give more comparable data to the current practice of giving tPA.

\section{Gender differences}

The finding by Amiri-Nikpour et $a 1 .{ }^{53}$ raises a question of whether minocycline is beneficial in both males and females. The epidemiology of ischemic stroke is sexually dimorphic-- ischemic stroke occurs more often in men than women, regardless of ethnicity. ${ }^{57}$

Estrogen has been shown to be neuroprotective in the brain. ${ }^{58}$ Female animals suffered less damage from acute stroke than males, though this could be reversed through ovariectomy. Male rats given estrogen had smaller infarcts than untreated males. ${ }^{59} \mathrm{~A}$ review by Bushnell examines the effect of hormones in female stroke. ${ }^{60}$ Yet, women continue to suffer fewer strokes than men in old age when they are post-menopausal, so this steroidal effect can't be the single factor mediating this difference. ${ }^{3}$

A review by Lang \& McCullough ${ }^{61}$ examined another factor regarding the effect of gender. Their findings suggest that the gender effect in treatment outcomes result from gender differences in caspase- 3 and cytochrome c activation as well as the role of PARP1 in both sexes. Caspase-dependent pathways were more harmful in females than males.

These findings might have affected the outcome of the AmiriNikpour et al. ${ }^{53}$ study that found no significant effect of administration of minocycline in females. However, this study was not placebo controlled and the sample size was small. No other clinical trials reported a difference between genders. Gender differences in treatment response will need to be addressed in future trials.

\section{Combining minocycline with tPA}

Using minocycline with tPA as a combination therapy may be expected to yield synergistic effects. tPA's use is worrisome due to its increased risk of subsequent hemorrhagic transformation. Minocycline can help in this area. One study has shown that after permanent focal ischemia, a PARP inhibitor reduced hemorrhagic transformation. ${ }^{62}$ More studies have been done on minocycline inhibition of MMPs in this regard.

Another way that minocycline could complement tPA therapy is by inhibition of MMPs. MMPs play a mediating role in the hemorrhagic transformation post-ischemic stroke.
Minocycline has been shown to decrease plasma MMP-9 levels in multiple studies. ${ }^{63,64} \mathrm{In}$ fact, an analysis of the Fagan et al. ${ }^{21}$ study showed that lower MMP-9 levels were found in those treated with tPA and minocycline. ${ }^{65}$

Minocycline doesn't affect the fibrinolytic and amidolytic properties of tPA. ${ }^{63}$ Additionally, minocycline was able to extend tPA's time window to 6 hours in an embolic focal ischemia model in rats with similar infarct volumes to tPA treatment at 1 hour postocclusion (and was also protective without tPA at 4 hours). ${ }^{64}$ Even in a type-1 diabetic rat model of focal embolic stroke, combination therapy of minocycline and tPA was more effective at reducing brain infarction, hemorrhagic transformation and hemispheric swelling after 24 hours. ${ }^{66}$

\section{Future prospects: replicating the NINDS study}

In 1995, the National Institute of Neurological Disorders and Stroke (NINDS) stroke trial was published, and it has since been credited as one of the most crucial final steps in getting tPA FDA approved in $1996 .{ }^{67}$

Replicating the methods and structure of the NINDS stroke trial with minocycline should yield the definitive data needed to prove minocycline's clinical effectiveness. Previous studies have had limited patient numbers, so achieving a larger patient pool, closer to three or four hundred, should be appropriate. It would be important to identify the effect of minocycline alone and a combination of minocycline and tPA. A $3 \mathrm{mg} / \mathrm{kg}$ dose of minocycline should be given intravenously and acutely for treatment, along with maintenance doses for 5 days; or, a $200 \mathrm{mg}$ dose of oral minocycline given in the same was as done in the previous studies. Furthermore, there will need to be subgroup analysis to determine if there are effects due to gender, stroke onset to treatment time, effectiveness of combination therapy, and age. Similar outcome measures should be used to the previous clinical studies on minocycline --the BI, mRS, NIHSS on days 1, 7, 30, and 90. A statistically significant improvement in these outcome measures should persuade the medical community to reconsider minocycline as an acute stroke therapy. The effect of minocycline in hemorrhagic strokes may have to be studied in a separate study.

A study of the effect of administration of minocycline to patients with ischemic stroke is in progress. ${ }^{68}$ The purpose of the study is to find out if the risk of ischemic strokes to convert into hemorrhagic strokes is reduced from administration of minocycline.

\section{Conclusion}

Minocycline therapy in acute ischemic stroke is promising. Minocycline is a well-tolerated drug that has been used for decades as an antibiotic. Its side effects are well-known and few. While the exact direct or indirect pathway in its neuroprotection has not been clearly illustrated as of yet, it seems to have multiple beneficial effects including PARP-1 inhibition, anti-apoptotic properties, scavenging of ROS, inhibition of microglia, and suppression of MMPs. The latter is especially important, as MMPs are important factors mediating hemorrhagic transformation and upregulated in tPA use.

As seen from the results of the five published clinical trials on minocycline in acute stroke, minocycline aids stroke recovery even when given up to 24 hours after stroke onset. Therefore, combining tPA with minocycline for acute treatment of stroke seems promising and could even possibly extend tPA's time window. However, previous clinical studies had important limitations. A future study should be 
done to illuminate minocycline's neuroprotection in a large, doubleblinded, placebo-controlled study in which minocycline is given IV in a time-efficient manner with or without tPA that also analyzes differences in gender, age, stroke onset to treatment time, age, and beneficiary effect in combination treatment, including possible extension of tPA window.

With the present knowledge about the efficacy of minocycline in reducing the effects of ischemic strokes the authors of the West Australian study known as the Intravenous Minocycline and TPA Stroke Study (WAIMATSS) ${ }^{68}$ concluded that: "Minocycline has practical advantages, including its well-established track record of safety (when used as an antibiotic), bioavailability (including orally), and the fact that it can be safely used in patients with hemorrhagic stroke. This makes it a candidate for use in the prehospital setting, without the need for imaging prior to administration. Ease of use and inexpensiveness also make it a candidate for widespread use without concomitant thrombolytic therapy in nontertiary, rural, and even third world settings. ${ }^{69}$ To reach that conclusion the authors point to the side effects and poor efficacy of the FDA approved treatment using administration of tPA and instead point to the advantages of the use of minocycline.

\section{Acknowledgments}

None.

\section{Conflicts of interest}

None.

\section{References}

1. Go AS, Mozaffarian D, Roger VL, et al. Executive summary: heart disease and stroke statistics-2014 update: a report from the American Heart Association. Circulation. 2014;129(3):399-410.

2. Kernan WN, Ovbiagele B, Black HR, et al. Guidelines for the prevention of stroke in patients with stroke and transient ischemic attack: a guideline for healthcare professionals from the American Heart Association/ American Stroke Association. Stroke. 2014;45(7):2160-2236.

3. Rosamond W, Flegal K, Furie K, et al. Heart disease and stroke statistics - 2008 update: a report from the American Heart Association Statistics Committee and Stroke Statistics Subcommittee. Circulation. 2008;117(4):e25-e146.

4. Sidney S, Rosamond WD, Howard VJ, et al. National Forum for Heart Disease and Stroke Prevention. The "heart disease and stroke statistics - 2013 update" and the need for a national cardiovascular surveillance system. Circulation. 2013;127(1):21-23.

5. Romero J, Wolf P. Epidemiology of stroke: legacy of the Framingham heart study. Glob Heart. 2013;8(1):67-75.

6. Mass M, Safdieh J. Ischemic stroke: pathophysiology and principles of localization. Hospital Physician Board Review Manual. 2009;13(1):1-5.

7. Gotoh O, Asano T, Koide T, et al. Ischemic brain edema following occlusion of the middle cerebral artery in the rat. I: The time courses of the brain water, sodium and potassium contents and blood-brain barrier permeability to 125I-albumin. Stroke. 1985;16(1):101-109.

8. Chamorro A, Hallenbeck J. The Harms and benefits of inflammatory and immune responses in vascular disease. Stroke. 2006;37(2):291-293.

9. Wang Q, Tang X, Yenari M. The inflammatory response in stroke. $J$ Neuroimmunol. 2007;181(1-2):53-68.

10. Wang $\mathrm{X}$, Zhu S, Drozda M, et al. Minocycline inhibits caspaseindependent and -dependent mitochondrial cell death pathways in models of Huntington's disease. Proc Natl Acad Sci USA. 2003;100(18):10483-10487.
11. Cronin C, Sheth K, Zhao X, et al. Adherence to Third European Cooperative Acute Stroke Study 3- to 4.5-hour exclusions and association with outcome: data from Get with the Guidelines-Stroke. Stroke. 2014;45(9):2745-2749.

12. Tissue Plasminogen Activator for Acute Ischemic Stroke. The National Institute of Neurological Disorders and Stroke rt-PA Stroke Study Group. N Engl J Med. 1995;333(24):1581-1518.

13. Collen D, Lijnen H. Thrombolytic agents. Thromb Haemost. 2005;93(4):627-630.

14. Vivien D, Gauberti M, Montagne A, et al. Impact of tissue plasminogen activator on the neurovascular unit: from clinical data to experimental evidence. J Cereb Blood Flow Metab. 2001;31(11):2119-2134.

15. Su E, Fredriksson L, Geyer M, et al. Activation of PDGF-CC by tissue plasminogen activator impairs blood-brain barrier integrity during ischemic stroke. Nat Med. 2008;14(7):731-737.

16. Lapchak P, Chapman D, Zivin J. Metalloproteinase inhibition reduces thrombolytic (tissue plasminogen activator)-induced hemorrhage after thromboembolic stroke. Stroke. 2000;31(12):3034-3040.

17. Nicole O, Docagne F, Ali C, et al. The proteolytic activity of tissueplasminogen activator enhances NMDA receptor-mediated signaling. Nature Med. 2001;7(1):59-64.

18. Duggar B. Aureomycin: a product of the continuing search for new antibiotics. Ann NY Acad Sci. 1948;51(Art. 2):177-181.

19. Saivin S, Houin G. Clinical pharmacokinetics of doxycycline and minocycline. Clin Pharmacokinet. 1988;15(6):355-366.

20. Fagan S, Cronic L, Hess D. Minocycline Development for Acute Ischemic Stroke. Transl Stroke Res. 2011;2(2):202-208.

21. Fagan S, Waller J, Nichols F, et al. Minocycline to improve neurologic outcome in stroke (MINOS): a dose-finding study. Stroke. 2010;41(10):2283-2287.

22. Susan S, Daugas E, Ravagnan L, et al. Two distinct pathways leading to nuclear apoptosis. J Exp Med. 2000;192(4):571-580.

23. Wang J, Wei Q, Wang C, et al. Minocycline Up-regulates Bcl-2 and Protects against Cell Death in Mitochondria. J Biol Chem. 2004;279(19):19948-19954.

24. Matsukawa N, Yasuhara T, Hara K, et al. Therapeutic targets and limits of minocycline neuroprotection in experimental ischemic stroke. BMC Neurosci. 2009;10:126.

25. Yellon D, Hausenloy D. Myocardial reperfusion injury. $N$ Engl J Med. 2007;357(11):1121-1135.

26. Kraus R, Pasieczny R, Lariosa-Willingham K, et al. Antioxidant properties of minocycline: neuroprotection in an oxidative stress assay and direct radical-scavenging activity. J Neurochem. 2005;94(3):819827

27. Domercq M, Matute C. Neuroprotection by tetracyclines. Trends Pharmacol Sci. 2004;25(12):609-612.

28. Tikka T, Fiebich B, Goldsteins, G, et al. Minocycline, a tetracycline derivative, is neuroprotective against excitotoxicity by inhibiting activation and proliferation of microglia. J Neurosci. 2001;21(8):2580 2588 .

29. Yenari MA, Xu L, Tang $\mathrm{XN}$, et al. Microglia potentiate damage to blood-brain barrier constituents: improvement by minocycline in vivo and in vitro. Stroke. 2006;37(4):1087-1093.

30. Yang Y, Salayandia V, Thompson J, et al. Attenuation of acute stroke injury in rat brain by minocycline promotes blood-brain barrier remodeling and alternative microglia/macrophage activation during recovery. J Neuroinflammation. 2015;10:26.

31. Rosenberg L, Louik C, Shapiro S. Nonsteroidal antiinflammatory drug use and reduced risk of large bowel carcinoma. Cancer. 1998;82(12):2326-2333 
32. Rosenberg G, Kornfeld M, Estrada E, et al. TIMP-2 reduces proteolytic opening of blood-brain barrier by type IV collagenase. Brain Res. 1992;576(2):203-207.

33. Asahi M, Wang X, Mori T, et al. Effects of matrix metalloproteinase-9 gene knock-out on the proteolysis of blood-brain barrier and white matter components after cerebral ischemia. J Neurosci. 2001;21(19):77247732 .

34. Jickling G, Liu D, Stamova B, et al. Hemorrhagic transformation after ischemic stroke in animals and humans. $J$ Cereb Blood Flow Metab. 2014;34(2):185-199.

35. Castellanos M, Leira R, Serena J, et al. Plasma metalloproteinase-9 concentration predicts hemorrhagic transformation in acute ischemic stroke. Stroke. 2003;34(1):40-46.

36. Montaner J, Molina C, Monasterio J, et al. Matrix metalloproteinase-9 pretreatment level predicts intracranial hemorrhagic complications after thrombolysis in human stroke. Circulation. 2003;107(4):598-603.

37. Rosell A, Ortega-Aznar A, Alvarez-Sabín J, et al. Increased brain expression of matrix metalloproteinase-9 after ischemic and hemorrhagic human stroke. Stroke. 2006;37(6):1399-1406.

38. Machado L, Kozak A, Ergul A, et al. Delayed minocycline inhibits ischemia-activated matrix metalloproteinases 2 and 9 after experimental stroke. BMC Neurosci. 2006;7:56.

39. Liu R, Yuan H, Yuan F, et al. Neuroprotection targeting ischemic penumbra and beyond for the treatment of ischemic stroke. Neurological Research. 2012;34(4):331-337.

40. Yrjänheikki J, Tikka T, Keinänen R, et al. A tetracycline derivative, minocycline, reduces inflammation and protects against focal cerebral ischemia with a wide therapeutic window. Proc Natl Acad Sci U S A. 1999;96(23):13496-13500.

41. Yrjänheikki J, Keinänen R, Pellikka M, et al. Tetracyclines inhibit microglial activation and are neuroprotective in global brain ischemia. Proc Natl Acad Sci U S A. 1998;95(26):15769-15774.

42. Power C, Henry S, Del Bigio M, et al. Intracerebral hemorrhage induces macrophage activation and matrix metalloproteinases. Ann Neurol. 2003;53(6):731-742.

43. Szymanska A, Biernaskie J, Laidley D, et al. Minocycline and intracerebral hemorrhage: influence of injury severity and delay to treatment. Exp Neruol. 2006;197(1):189-196.

44. Xu L, Fagan S, Waller J, et al. Low dose intravenous minocycline is neuroprotective after middle cerebral artery occlusion-reperfusion in rats. BMC Neurol.4:7

45. Hewlett K, Corbett D. Delayed minocycline treatment reduces longterm functional deficits and histological injury in a rodent model of focal ischemia. Neuroscience. 2006;141(1):27-33.

46. Liu Z, Fan Y, Won S, et al. Chronic treatment with minocycline preserves adult new neurons and reduces functional impairment after focal cerebral ischemia. Stroke. 2007;38(1):146-152.

47. Colovic M, Caccia S. Liquid chromatographic determination of minocycline in brain-to-plasma distribution studies in the rat. $J$ Chromatogr B Analyt Technol Biomed Life Sci. 2003;791(1-2):337-343.

48. Coskey R. Acne: treatment with minocycline. Cutis. 1976;17(4):799801.

49. Clark B, Hodgson C, Dornbush A, et al. Minocycline administered intravenously: pharmacological activity and clinical experience Curr Ther Res Clin Exp. 1974;16(9):865-877.

50. Lampl Y, Boaz M, Gilad R, et al. Minocycline treatment in acute stroke: an open-label, evaluator-blinded study. Neurology. 2007;69(14):1404 1410 .
51. Padma Srivastava MV, Bhasin A, Bhatia R, et al. Efficacy of minocycline in acute ischemic stroke: a single-blinded, placebo-controlled trial. Neurol India. 2012;60(1):23-28.

52. Kohler E, Prentice D, Bates T, et al. Intravenous Minocycline in Acute Stroke: A Randomized, Controlled Pilot Study and Meta-analysis. Stroke. 2013;44(9):2494-2499.

53. Amiri-Nikpour M, Nazarbaghi S, Hamdi-Holasou M, et al. An openlabel evaluator-blinded clinical study of minocycline neuroprotection in ischemic stroke: gender-dependent effect. Acta Neurol Scad. 2015;131(1):45-50.

54. Jonas M, Cunha B. Minocycline. Ther Drug Monit. 1982;4(2):137-145.

55. Carney S, Butcher R, Dawborn J, et al. Minocycline excretion and distribution in relation to renal function in man. Clin Exp Pharmacol Physiol. 1974;1(4):299-308.

56. Hrobjartsson A, Gotzsche P. Is the placebo powerless? An analysis of clinical trials comparing placebo with no treatment. $N$ Engl J Med. 2001344(21):1594-1602.

57. Sudlow C, Warlow C. Comparable studies of the incidence of stroke and its pathological types: results from an international collaboration. International Stroke Incidence Collaboration. Stroke. 1997;28:491-499.

58. Brann DW, Dhandapani K, Wakade C, et al. Neurotrophic and neuroprotective actions of estrogen: basic mechanisms and clinical implications. Steroids. 2007;7(5):381-405.

59. McCullough L, Alkayed N, Traystman R, et al. Postischemic estrogen reduces hypoperfusion and secondary ischemia after experimental stroke. Stroke. 2001;32(3):796-802.

60. Bushnell CD. Stroke and the female brain. Nat Clin Pract Neurol. 2008;4(1):22-33.

61. Lang J, McCullough L. Pathways to ischemic neuronal cell death: are sex differences relevant? J Transl Med. 2008;6:33.

62. Haddad M, Beray-Berthat V, Coqueran B, et al. Reduction of hemorrhagic transformation by $\mathrm{PJ} 34$, a poly(ADP-ribose)polymerase inhibitor, after permanent focal cerebral ischemia in mice. Eur $J$ Pharmacol. 2008;588(1):52-57.

63. Machado L, Sazonova I, Kozak A, et al. Minocycline and tissue-type plasminogen activator for stroke: assessment of interaction potential. Stroke. 2009;40(9):3028-3033.

64. Murata Y, Rosell A, Scannevin R, et al. Extension of the thrombolytic time window with minocycline in experimental stroke. Stroke. 2008;39(12):3372-3377.

65. Switzer J, Hess D, Ergul A, et al. Matrix metalloproteinase-9 in an exploratory trial of intravenous minocycline for acute ischemic stroke. Stroke. 2011;42(9):2633-2635.

66. Fan X, Lo E, Wang X. Effects of minocycline plus tissue plasminogen activator combination therapy after focal embolic stroke in type 1 diabetic rats. Stroke. 2013;44(3):745-752.

67. Bruce N, Neil W, Zivin J. Medico-legal aspects of using tissue plasminogen activator in acute ischemic stroke. Curr Treat Options Cardiovasc Med 2011. 2011;13(3):233-239.

68. Blacker D, Prentice D, Alvaro A, et al. Reducing haemorrhagic transformation after thrombolysis for stroke: a strategy utilising minocycline. Stroke Research and Treatment. 2013;2013:362961.

69. Hess D, Howard V, Howard G. Minocycline: the search for a community hospital acute stroke therapy Neurology India. Neurology India. 2012;60(1):1-2. 